\title{
Photographie, Ethnographie und physische Anthropologie im 19. Jahrhundert: Ein Überblick für den deutschen Sprachraum
}

\author{
THOMAS THEYE \\ Bremen
}

VORBEMERKUNG: DIE BEGRIFFE

Im heutigen deutschen Sprachgebrauch beschäftigt sich die Ethnographie, wörtlich Völkerbeschreibung, mit der Beschreibung und Erforschung kultureller Erscheinungen. Ethnologie, im Wortsinne Völkerkunde, beginnt dagegen in methodologischer Hinsicht dort, wo "bewußt Erklärungen der Erscheinungen in systematisch zusammenhängender Form geliefert werden. [...] Die Erklärung von Verschiedenheiten und Übereinstimmungen in den Kulturen der Völker ist Ziel der Ethnologie" ". Diese Trennung zwischen Sammlung und Beschreibung einerseits und ihrer theoretischen Verarbeitung andererseits ist in einem kritischen Licht ${ }^{2} \mathrm{zu}$ sehen, weil sie vorgibt, Arbeitsbereiche zu trennen, die in Wirklichkeit auf das Engste miteinander verknüpft sind. Dennoch wird im gegenwärtigen Sprachgebrauch einem eher beschreibenden ein eher systematisch auswertender und theoretischvergleichender Arbeitsschritt gegenübergestellt. Die Verwendung der Begriffe Ethnographie und Ethnologie folgt daher auch im vorliegenden Text diesem Grundsatz.

\footnotetext{
1 Fischer (1983: 12).

2 Dies gilt für explizit festgelegte Beobachtungsanleitungen, die aufgrund eines bestimmten wissenschaftlichen, eben ethnologischen, Interesses, bestimmte Aspekte anderer Kulturen als beobachtenswert definieren und andere hingegen nicht. Aber auch das Auge des Forschungsreisenden unterliegt bestimmten, zum Teil unbewußt getroffenen Vorentscheidungen, die sein Beobachtungsfeld eingrenzen. Schließlich ist ajede Ethnographie als Beschreibung der Lebensweise eines Volkes immer auch Vergleich. Vergleich der beobachteten mit der eigenen Lebensweise des Ethnographen. Festgestellt werden Unterschiede und Ähnlichkeiten". Fischer (1983: 12). Vgl. ebenso Kohl (1993: 99). Vor diesem Hintergrund stellt Hildebrandt (1994: 352f.) nachdrücklich die Neutralität beobachteter "Fakten" in Frage und fordert, die jeder Beobachtung zu Grunde liegenden Kategorien offenzulegen und in die Analyse miteinzubeziehen. Handele es sich um historische ethnographische Arbeiten, so sei eine Untersuchung des jeweiligen Kontextes unabdingbar.
} 
Neuere Forschungen ${ }^{3}$ des Leidener Historikers Han F. Vermeulen führen die Begriffe Ethnographie und Völkerkunde auf eine Publikation des Göttinger Historikers und Slawisten August Ludwig Schlözer (1735-1809) aus dem Jahre 1771 zurück. Nur kurze Zeit später finden sich beide Begriffe ebenfalls bei dem Göttinger Historiker Johann Christoph Gatterer (1727-1799). Der Terminus Ethnologie wurde hingegen 1783 von dem slowakischen Historiker Adam František Kollár (1723-1783) in Wien und 1787 von dem Schweizer Theologen Alexandre-César Chavannes (1731-1800) in Lausanne geprägt ${ }^{4}$.

Der Begriff Anthropologie als, im Wortsinne, allgemeine Wissenschaft vom Menschen, wurde 1501 von dem Magdeburger Arzt und Geistlichen Magnus Hundt (1449-1519) erstmals verwendet. Die Anthropologie des 19. Jahrhunderts befaßte sich im deutschen Sprachraum aber vornehmlich mit dem körperlichen Erscheinungsbild des Menschen. Diese physische Anthropologie ist mit dem Berliner Pathologen und Anthropologen Rudolf Virchow (1821-1902) als die "Erforschung und Darstellung der physischen Eigenschaften des Menschen" 5 zu verstehen. Sie verfolgte das Ziel, eine Vielzahl menschlicher Körpermerkmale zu unterscheiden und einzuteilen, um so die menschliche Entwicklungsgeschichte im biologischen Sinne zu erhellen.

Während sich die physischen Anthropologie damals wie heute mit allen Angehörigen des Menschengeschlechtes befaßt, gibt es zum "Forschungsgegenstand" der Ethnographie gegenwärtig durchaus unterschiedliche Auffassungen: Entweder werden kulturelle Erscheinungen aller Völker eingeschlossen und thematisiert, oder aber nur diejenigen bestimmter Ethnien auf Grund mehr oder weniger willkürlicher Kriterien (fremd, überseeisch, schriftlos, vorindustriell; Naturvölker, Stammeskulturen etc.) zum "Forschungsgebiet" erklärt.

Für das 19. Jahrhundert beinhaltete der Begriff Ethnographie im deutschen Sprachraum dagegen etwas anderes: Diese Teildisziplin der Geographie beschäftigte sich lange Zeit mit allen Völkern des Erdenrundes. Hierzu zählten sowohl Untersuchungen der körperlichen Erscheinungsform des Menschen, als auch das Studium der Sitten und Gebräuche oder des materiellen Kulturinventars. Ethnographische Standardwerke, wie etwa die Kompilationen Friedrich Müllers, Allgemeine Ethnographie, 1873, und Oscar Peschels Völkerkunde, 1874, behandelten so auch beide Gebiete. Leitender Gesichtspunkt in der Gliederung der darzustellenden Völker und Kulturen

\footnotetext{
3 Vermeulen (1995: 42-48).

4 Streck (1987: 10).

5 Virchow (1875: 571).
} 
war zum einen die Einteilung in verschiedene Menschenrassen, zum anderen die Konstruktion evolutionistischer Stufenleitermodelle.

\section{HERDERS "ANTHROPOLOGISCHER WUNSCH"}

Es wäre schön, wenn ich jetzt durch eine Zauberruthe alle bisher gegebnen unbestimmten Wortbeschreibungen in Gemählde verwandeln und dem Menschen von seinen Mitbrüdern auf der Erde eine Gallerie gezeichneter Formen und Gestalten geben könnte. Aber wie weit sind wir noch von der Erfüllung dieses anthropologischen Wunsches!

Indeß $\mathrm{da}$ in den neuesten Zeiten der edle Bemerkungsgeist auch für unser Geschlecht wirklich schon erwacht ist und man von einigen, wie wohl nur von wenigen Nationen Abbildungen hat, [...] so wäre es ein schönes Geschenk, wenn Jemand, der es kann, die hie und da zerstreueten treuen Gemälde der Verschiedenheit unsres Geschlechts sammlete [... $]^{6}$.

Mit diesen Worten schloß Johann Gottfried Herder (1744-1803) 1785 sein sechstes Buch der Ideen zur Philosophie der Geschichte der Menschbeit (1784-1791). Zuvor hatte er darin die verschiedenartige körperliche Gestalt und die Physiognomien der Völker beschrieben, die das Erdenrund bewohnten. Herder verlieh in diesem Schlußwort dem Wunsch nach mehr und vor allem nach authentischen Bilddokumenten der Erscheinungsformen des Menschen besonderen Nachdruck. Die Erfüllung seines Wunsches schien den Zeitgenossen in greifbare Nähe gerückt, als die Photographie die Szene betrat.

\section{BLUMENBACHS ABBILDUNGEN NATURHISTORISCHER GEGENSTÄNDE}

Ein Zeitgenosse der Aufklärungszeit, der Göttinger Naturforscher und Anthropologe Johann Friedrich Blumenbach (1752-1840), sollte sich Herders Desideratum nur kurze Zeit später zu eigen machen. Auch Blumenbach äußerte berechtigte Kritik an seinen bildnerischen Vorgängern. Er beklagte im Vorwort seiner hundert Tafeln umfassenden und von 1796 an in zehn Heften publizierten Abbildungen naturbistorischer Gegenstände, daß ihm kein "anthropologisches, oder überhaupt naturhistorisches Werk bekannt ist, worin ächte, porträtmässige und characteristische Abbildungen der wichtigsten Rassen im Menschengeschlecht enthalten wären" ${ }^{7}$. In den

6 Herder ([1785] 1967:250).

7 Blumenbach (1796: o.S.). 
"Völker-Gallerien" und Trachtenbüchern seiner Zeit sah Blumenbach die Porträts "Von wilden Völkerschaften fremder Welttheile, was die Gesichtsbildung betrifft, bloss aufs willkührliche Gerathewohl hingeworfen" ${ }^{8}$. Eine solche Schelte der frühen Bildquellen läßt sich durch vielfältige Belege dokumentieren - kritische Stimmen, die insbesondere im 19. Jahrhundert laut wurden. Der aus Gießen stammende Genfer Zoologe und Geologe Carl Vogt (1817-1895) behandelte in seinen 1863 veröffentlichten Vorlesungen über den Menschen, seine Stellung in der Schöpfung und in der Geschichte der Erde auch verschiedene Abbildungsverfahren in ihrem Nutzen für die physische Anthropologie 9: Er maß dabei den "meisten Rassenbildern, die bis in die neueste Zeit geliefert wurden, [...] nur einen höchst geringen oder selbst gar keinen Werth" ${ }^{10}$ bei: "Was also Lebensbilder von Rassen betrifft, so darf man kühn behaupten, daß die meisten derselben dem Zwecke einer ernsten Forschung nicht entsprechen, sondern im Gegentheile nur dazu dienen, das Verständniß irre zu leiten und auf untergeordnete Punkte zu lenken" "11.

Die Kritik richtete sich vornehmlich darauf, daß sich die hiesigen Künstler und Illustratoren in der Darstellung fremder Menschen und Völker zu stark von ihren europäischen und zumeist klassizistischen Vorstellungen vom Aussehen des menschlichen Körpers leiten ließen. Hierdurch erschienen dem Berliner Anatomen, Physiologen und Anthropologen Gustav Fritsch (1838-1927), der sich intensiv mit den Anwendungsmöglichkeiten der Photographie befaßte, "die illustrirten Reisewerke des späteren Mittelalters bis in die neuere Zeit hinsichtlich der Wiedergabe von Volkstypen bis zur Lächerlichkeit unbrauchbar" ${ }^{12}$.

8 Blumenbach (1796:o.S.).

9 Die Überlegungen Vogts sind nicht nur wegen ihres Bekenntnisses zur Treue der photographischen Wiedergabe bedeutsam - darüber hinaus stellen sie für den deutschen Sprachraum eine der frühesten Anleitungen zum wissenschaftlichen Gebrauch der Photographie in der physischen Anthropologie dar. Ginge es dem Forscher um exakte Vergleichbarkeit und das nachträgliche Ausmessen des abgebildeten Objektes, zum Beispiel eines Schädels, so empfahl Vogt (1. 1863: 87) die geometrische Zeichnung mit Hilfe eines Zeichengerätes. Dagegen sah er in der Photographie "eines der unschätzbarsten Hilfsmittel» (1. 1863: 84), wenn es gälte, zum Zwecke bildlicher Erläuterung, charakteristische Merkmale lebender Menschen perspektivisch abzubilden. Vgl. Vogt (1. 1863: 86 und 89).

10 Vogt (1. 1863: 83).

11 Vogt (1. 1863: 84)

12 Fritsch (1881:[212]). 


\section{PHOTOGRAPHIEN ALS "MATERIELLER ANHALT"}

Der anscheinend nicht von der Hand des Photographen kontrollierte Detailreichtum photographischer Aufnahmen, die Genauigkeit der Kopie eines gewählten Wirklichkeitsausschnittes und die Möglichkeiten einer enzyklopädischen Inventarisierung fügten sich nahtlos in die Zielvorstellungen eines positivistischen Wissenschaftsverständnisses ein, das eine detailtreue Dokumentation der sichtbaren Welt ${ }^{13}$ anstrebte. Als "Hauptregel" für den Reisenden galt es "Material zu sammeln, das später in Musse von ihm selbst oder von anderen wissenschaftlich bearbeitet werden soll. Er hat Thatsachen zu beobachten und darzustellen" ${ }^{14}$. Nach dem Leitmotiv, "Wir wollen nicht glauben, sondern schauen" ${ }^{15}$, hätte dann der heimische Leser oder Betrachter anhand der Vielzahl wiedergegebener Details genauso urteilen können wie der Reisende am Orte des Geschehens selbst: "Der Reisende möge uns genau sagen, was er sieht, wir werden selbst ahnen, was daraus folgt, ${ }^{16}$.

Unter diesen Umständen stellte die Photographie das adäquate wissenschaftliche Dokumentationsmittel dar, da sie Fritsch zufolge eben jene positiven Belege lieferte, "welche als materieller Anhalt dem Unkundigen die directe Anschauung zu ersetzen vermögen und gleichzeitig als Correctiv für die subjective Auffassung des Reisenden dienen können ${ }^{17}$. So machte die physische Anthropologie im 19. Jahrhundert aus der Sicht der Zeitgenossen durch die untrüglichen, photographischen "Beweise", die anscheinend erst jetzt die genaue Beschreibung und den Vergleich fremder Völker ermöglichten, den entscheidenden Sprung hin zu einer vollgültigen naturwissenschaftlichen Disziplin. Der Anthropologie wurde, wie Fritsch

13 Auch die, vom späteren Direktor der Deutschen Seewarte in Hamburg, Georg von Neumayer, erstmals im Jahre 1875 herausgegebene Anleitung zu wissenschaftlichen Beobacbtungen auf Reisen folgte diesem Ziel. Mit ihrer Hilfe sollte die seit der Gründung des Deutschen Reichs immer größer werdende Zahl von Reisenden und Residenten in Übersee zu nutzbringenden Beobachtungen für die heimischen "Schreibtischgelehrten" angeregt werden. Das Werk Neumayers erlebte drei Auflagen: 1875, 1888 und 1906. Der Rahmen der vorliegenden Darstellung läßt ein näheres Eingehen auf die verschiedenen Beobachtungsanleitungen nicht zu. Vgl. zu diesem Thema meine Darstellung im Katalogbuch Der geraubte Schatten (1989: 72, 89f.).

14 Aus dem Kapitel "Linguistik" des Sprachwissenschaftlers Heymann (Heinrich) Steinthal in: Neumayer (1875: 551).

15 Meitzen (1875: 154).

16 Meitzen (1875: 154).

17 Fritsch (1875: 605). 
es formulierte, "die Photographie [zur] hilfreichen Ariadne, welche den aus dem unendlichen Wirrsal falscher Vorstellungen herausleitenden, die Anschauungen befreienden Faden lieferte" ${ }^{18}$.

\section{FRÜHE ANTHROPOLOGISCHE PHOTOGRAPHIEN}

Der Pariser Photograph E. Thiésson ${ }^{19}$ photographierte im Sommer 1844 zwei in Paris weilende Botokuden, indianische Einwohner des nordöstlichen Brasiliens, eine Frau und einen Mann. Die dabei entstandenen fünf Daguerreotypien wurden am 2. September 1844 der Akademie der Wissenschaften durch ihren Präsidenten, Etienne-Renaud-Augustin Serres (1787-1868), vorgelegt. Dabei lobte die Akademie "die Reinheit jener [photographischen] Abzüge sowie auch die Exaktheit, mit der die Eigentümlichkeiten wiedergegeben sind, welche jene Menschenvarietät auszeichnen" ${ }^{20}$.

Für den deutschen Sprachraum läßt sich gegenwärtig keine ähnlich frühe wissenschaftliche Nutzung der Photographie nachweisen. Erst als die Brüder Schlagintweit im Auftrage der britischen East India Company von 1854 bis 1857 Indien und die Himalaja-Region bis hinein nach Zentralasien bereisten, sollte Robert Schlagintweit ${ }^{21}$ (1833-1885) neben Gipsabformungen und Körpermaßen auch anthropologische Photographien aufnehmen. Die Resultate der auf besondere Vermittlung Alexander von Humboldts ${ }^{22}$ (1769-1859) zustandegekommenen Forschungsreise bestanden in Untersuchungen des Erdmagnetismus', in Höhenmessungen und kartographischen Arbeiten sowie in meteorologischen Beobachtungen. Das an Humboldt gemahnende universale Forschungsinteresse der Reisenden richtete sich daneben auf botanische, zoologische wie auch auf ethnographische Beobachtungen und Sammlungen sowie auf die physische Anthropologie des indischen Subkontinents.

Als weiterer Forschungsreisender, der früh mit photographischen Mitteln über die bereisten Länder, Völker und Kulturen berichtete, ist der Berli-

18 Fritsch (1911: 18)

19 Auf die Bedeutung Thiéssons für die anthropologische Photographie hat besonders Hartmut Krech (1984: 9f.) hingewiesen. Ihm sind auch die Angaben zur Entstehung der ersten anthropologischen Photographien zu danken.

20 Serres (1844: 490). Übersetzung Theye. Im Original heißt es: "Il fait remarquer la netteté de ces épreuves, ainsi que l'exactitude avec laquelle sonts reproduits les caractères qui distinguent cette variété humaine».

21 Vgl. Körner (1989: 310-320)

22 Vgl. Kick (1982: 75f.). 
ner Ethnograph und Forschungsreisende Fedor Jagor (1816-1900) zu erwähnen. Auf seiner Reise nach Südostasien in den Jahren 1857 bis 1861 photographierte Jagor vornehmlich topographische Ansichten. Zum Porträtieren verwendete er hingegen zumeist die Camera lucida als Zeichenhilfe. Aus dem Kreis der Berliner Anthropologen ist besonders auf Fritsch zu verweisen, der das südliche Afrika in den Jahren 1863 bis 1866 bereiste und dort eine umfangreiche Porträtsammlung aufnahm. Auf ihn gehen die Hinweise zur Anfertigung anthropologischer Photographien zurück, wie sie dem Standardtext für den Gebrauch der Photographie auf Forschungsreisen zu entnehmen sind: "Praktische Gesichtspunkte für die Verwendung zweier dem Reisenden wichtigen technischen Hülfsmittel: das Mikroskop und der photographische Apparat”. Der Text ist in der 1875 durch den Ozeanographen, Geophysiker und Meteorologen Georg Balthasar von Neumayer (1826-1909) herausgegebenen Anleitung zu wissenschaftlichen Beobachtungen auf Reisen ${ }^{23}$ enthalten.

Fritsch führte eine systematische Gliederung für Photographien von Menschen ${ }^{24}$ ein und forderte dabei eine strikte Aufteilung der photographischen Dokumentation des Fremden. Priorität sollten "physiognomische" ${ }^{25}$ Aufnahmen haben, die, begleitet von einem Maßtab ${ }^{26}$, die körperliche Gestalt des

23 Die Photographie als Aufzeichnungsverfahren im Dienste der physischen Anthropologie und Ethnographie hatte für den deutschen Sprachraum zum ersten Male in den Ratbschläge für antbropologische Untersuchungen auf Expeditionen der Marine (1872: 28-29) nähere Erwähnung gefunden. Die dort noch sehr knapp gehaltenen und nicht namentlich ausgewiesenen Hinweise zur Anwendung der Photographie auf Forschungsreisen stammten mit Sicherheit aus der Feder von Fritsch, zeigen sie doch deutliche Übereinstimmungen hinsichtlich Gliederung, Inhalt und Diktion mit dessen späterem Aufsatz in Neumayers Anleitung $z u$ wissenschaftlichen Beobachtungen auf Reisen (1875: 591-625).

${ }^{24}$ Fritsch griff damit auf die entsprechende Aufgliederung der anthropologischen Aufnahmen" in "physiognomische" und "ethnographische" zurück, wie sie bereits in den Rathscblägen von 1872 vorgesehen war. Dort wurde zwischen "physiognomischen Aufnahmen, welche die physische Beschaffenheit der Völker darstellen" (1872: 28) und "ethnographischen Aufnahmen" (1872: 29) unterschieden.

25 Fritsch (1875: 606).

26 Der Maßstab tauchte im deutschen Sprachraum erstmals als Aufnahmegegenstand in den Ratbschlägen (1872: 28) und später bei Fritsch (1875: 608) auf. Bandmaß oder ähnliche Gerätschaftschaften sollten Anhaltspunkte für eine nachträgliche Vermessung des Aufgenommenen an Hand der Photographie liefern. Im angelsächsischen Raum war es John Lamprey, der 1869 sein System der anthropometrischen Photographie vorstellte. Hierbei wurde der Photographierte vor einem dunklen Stoffhintergrund aufgenommen. Auf diesen war mit feinen, weißen Seidenfäden eine Lineatur derart aufgetragen, daß sich Quadrate von je zwei Zoll Seitenlänge ergaben. Der Berliner Anatom und Anthropologe Robert Hartmann brachte in der Zeitschrift für Ethnologie (1869: 184) 
Menschen abbildeten. Demgegenüber standen "ethnographische ${ }^{27}$ Aufnahmen, welche die Lebensverhältnisse, Sitten und Gebräuche zeigten. Heute ist im Sinne der physischen Anthropologie von anthropologischen Photographien zu sprechen, denen die ethnographischen Photographien gegenüberzustellen sind. Die von Fritsch formulierten Aufnahmehinweise für anthropologische Photographien machte sich insbesondere der polnische Forschungsreisende Jan Stanislaw Kubary (1846-1896) zu eigen, der während der 1870er Jahre die Südsee für das Hamburger Handelshauses Joh. Ces. Godeffroy \& Sohn bereiste.

Nach dem Eindringen der großen westlichen Kolonialmächte in China und Japan um die Mitte des 19. Jahrhunderts suchten auch kleinere europäische Staaten ihre Chance, ein Stück aus dem kolonialen "Kuchen" zu erhaschen. Eine Expedition wie die Erdumsegelung der österreichischen Fregatte "Novara" in den Jahren 1857 bis 1859 zielten neben dem Aushandeln von Handelsverträgen auch darauf ab, ein wissenschaftliches Programm zu absolvieren, in dem unter anderem auch Anthropologie und Ethnographie Platz fanden. Mit von der Partie waren hier bereits des Photographierens Kundige, wie zum Beispiel der Geologe Ferdinand von Hochstetter (1829-1884), der Publizist Karl Ritter von Scherzer (1821-1903) oder der Photograph Bruno Hamel ${ }^{28}$. Mit einiger Bestimmtheit ist allerdings nur von dem Letztgenannten zu sagen, daß er seine photographischen Kenntnisse auch in die Tat umgesetzt hat.

An der "K. K. Mission nach Ostasien und Südamerika" in den Jahren 1868 bis 1870 nahm der Wiener Photograph Wilhelm Burger (1844-1920) als offizieller Expedionsphotograph teil ${ }^{29}$. Zu seinen Aufgaben gehörte die umfangreiche Dokumentation von kunsthandwerklichen Gegenständen für die heimischen Initiatoren der Expedition. Daneben ermöglichte er Einblicke in die japanische Gesellschaft zur Zeit der großen politischen und sozialen Umwälzungen der Meiji-Restauration.

einen Hinweis auf Lampreys Verfahren, wie auch einige seiner Photographien 1873/74 in Carl Dammanns Anthropologisch-Ethnologischem Album in Photographien Verwendung fanden.

27 Fritsch (1875: 606).

28 Vgl. Hochstetter (1863: XVII-XX), der Hamel als Photograph etlicher Abbildungen in in seinem Reisebericht, Neuseeland, ausweist. Zur "Novaran-Expedition und zu Scherzer siehe Theye (1995).

29 Wilhelm Burger nahm bis zum 31. Oktober 1869 an der offiziellen Expedition teil, mußte dann aber krankheitshalber in Yokohama aus dem Dienst scheiden. Nach Wiederherstellung seiner Gesundheit hielt er sich noch bis zum Anfang des Jahres 1870 in Japan auf und langte im Frühjahr 1870 wieder in Europa an. Vgl. Rosenberg (1984: 27f.). 
Mit den 60er Jahren des 19. Jahrhunderts begann überall auf der Erde die große Zeit der Photostudios, die in sogenannten Vertragshäfen, an Handelsplätzen und überall dort eröffnet wurden, wo sich westliche Kaufleute, Beamte, Militärs und andere im Gefolge des Kolonialismus niederließen. Dabei erlangten auch einige aus dem deutsch Sprachraum stammende Photographen Bedeutung wie etwa die Ateliers Richard Buchta in Khartum, K. Feilberg in Deli, Sumatra, E. Gsell in Saigon, Wilhelm Hammerschmidt in Kairo, G. R. Lambert \& Co. in Singapur, Baron Raimund von Stillfried-Ratenicz in Yokohama sowie John William Lindt in Grafton, New South Wales.

Photostudios verkauften neben der Darstellungen von Landschaft und Sehenswürdigkeiten in großem Umfange Genredarstellungen der Einheimischen. Diese sogenannten "native types" hielten den Habitus der fremden Menschen, ihr körperliches Erscheinungsbild, ihre Kleidung, Haartracht und Schmuck, Körperhaltung, aber auch gestellte "häusliche" Verrichtungen und gewerbliche Tätigkeiten fest. Vielfach bestand die Intention der Photographen darin, unter gezielter Ausblendung aller Attribute, die auf westliche Einflüsse hätten hindeuten können, ein vermeintlich ursprüngliches Leben zu inszenieren. Darüberhinaus nahmen erotische Inszenierungen einen bedeutenden Raum im kommerziellen Bildangebot ein. Zu den Abnehmern solcher Studioaufnahmen, die es selbst aus den entlegensten Winkeln der Erde gab, zählten zunächst die europäischen Residenten und Touristen, welche die Photographien als Souvenirs erwarben. Aber auch wissenschaftlich gebildete Forschungsreisende griffen gern auf das kommerzielle Bildangebot zurück, obwohl der ethnographische Wert der Studiophotographien bereits damals nicht unumstritten war. Sie kombinierten, wohl auch aus Einsicht in ihre eigenen technischen wie bildnerischen Unzulänglichkeiten, lange Zeit eigene Schnappschüsse mit den in Studios vertriebenen Photographien. Solche Mischformen setzten sich auch bis in ethnologische Werke hinein fort, wie etwa die in den Jahren 1885-1888 erschienene Völkerkunde des Leipziger Geographen und Ethnologen Friedrich Ratzel (1844-1904) belegt. Sie enthält in großer Zahl Illustrationen, die auf eine kommerzielle Bildproduktion zurückgehen.

\section{ZAHLLOSE ÜBERGÄNGE}

Der Photograph Thiésson sollte sich noch einmal auf anthropologischem Feld betätigen. Während einer Reise in der ersten Hälfte des Jahres 1845 entstanden in Lissabon und Cadiz zweiundzwanzig Aufnahmen ${ }^{30}$ von Afrika-

30 Anonymus (1845: 400). 
nerinnen und Afrikanern, die vermutlich aus den portugiesischen Kolonien stammten. Serres unterbreitete daraufhin am 21. Juli 1845 der Akademie die Ansichten einer Untersuchungskommission über "Observations sur l'application de la photographie à l'étude des races humaines" ${ }^{31}$. Darin wurde die Anwendung des neuen Mediums für ein "Musée photographique des races humaines ${ }^{32}$ vorgeschlagen, das den Menschen aus allen Teilen der Welt in seinen verschiedenen körperlichen Erscheinungsformen darstellen und vergleichen helfen sollte.

Die Nachricht von dieser neuen Anwendungsmöglichkeit des jungen Bildmediums drang augenscheinlich rasch nach Deutschland. Das in Stuttgart erscheinende Polytechnische Journal übermittelte noch 1845 in einer Miszelle mit dem Titel "Anwendung der Photographie zum Studium der Menschenraçen" ${ }^{33}$ eine Zusammenfassung der "Observations [...]" Serres'. Darin wurde die Möglichkeit hervorgehoben, daß durch die photographisch vermittelten "positiven Kenntnisse" ${ }^{34}$, sich große Reisen in entfernte Weltgegenden zum "Studium der Menschenraçen" ${ }^{35}$ entbehren ließen. Daneben läge der besondere wissenschaftliche Nutzen der Photographie darin, daß sie eine "vergleichende Betrachtung hinsichtlich der Race" ${ }^{36}$ ermöglichte: "Von mehreren Varietäten dieser äthiopischen Race sind auf den Lichtbildern die Uebergänge von der einen zur anderen auf das Deutlichste wahrzunehmen" ${ }^{37}$.

31 Serres (1845: 243-246). Auf die Bedeutung Serres' für die anthropologische Photographie hat besonders Krech (1984: 11f.) hingewiesen.

32 Serres (1845: 243).

33 So lautete der Titel einer nicht namentlich ausgewiesenen Miszelle aus dem Polytechnischen Journal 26. 1845, H. 17: 400, welche über die Photographien Thiéssons sowie ihre Präsentation durch Arago und Serres Auskunft gibt. Wolfgang Hesse, Dresden, machte mich freundlicherweise auf diese Textstelle aufmerksam. Das in Weimar erscheinende Journal für Malerei und bildende Kunst oder Mittbeilungen der neusten Erfabrungen und Verbesserungen in allen Zweigen der Malerei, der Bildhauerei, Daguerreotypie (Photographie), der Farbenkunde und Farbenchemie und die in diese Fächer einschlagende Bibliographie 2. 1845, H. 2: 59f., brachte im selben Jahr einen Bericht zum nämlichen Thema, der allerdings um einiges kürzer war als derjenige im Polytechnischen Journal. Ich danke Uwe Steen, Busdorf, für den Hinweis auf dieses Zitat.

34 Anonymus (1845: 400).

35 Anonymus (1845: 400)

36 Anonymus (1845: 400)

37 Anonymus (1845: 400). Wenn auch die hier verwendete Bezeichnung, "äthiopische Race», auf Blumenbach zurückgeht, so verwendete der Autor der Miszelle des Polytechnischen Journals jedoch den Begriff der Varietäten nicht wie Blumenbach für dessen fünf Hauptrassen, sondern in Anlehnung an Serres, als einen Unterbegriff für die verschiedenen Erscheinungsformen, die sich innerhalb einer Menschenrasse finden 
Die hier verwendete Bezeichnung "äthiopische Race" geht auf Blumenbach zurück, der an Hand ästhetisch begründeter Kriterien in der Betrachtung der Schädelformen seiner anatomischen Sammlung eine Einteilung der Menschheit in fünf Hauptvarietäten ${ }^{38}$ vorgeschlagen hatte. Wichtiger noch als dieses, lange Zeit ins 19. Jahrhundert hineinwirkende Einteilungsmodell, das sich auf eine umfangreiche, aber mehr oder weniger zufällig zusammengekommene Schädelsammlung stützte, ist jedoch die aufklärerische Einsicht Blumenbachs in die Einheit des Menschengeschlechtes und die Vielzahl kaum wahrnehmbarer und fließender Übergänge ${ }^{39}$ zwischen den von ihm vorgeschlagenen Hauptrassen.

lassen sollten. Blumenbach hatte hingegen die Menschenrassen in der biologischen Terminologie als Varietäten, Spiel- oder Abarten, als Untereinheit einer Art, als subspecies verstanden. Hinter diesen Unterschieden verbergen sich verschiedene Annahmen über den Ursprung des Menschengeschlechtes: Die Subsumierung aller Menschenrassen unter eine Art verweist auf eine monogenetische Annahme, die Einheit des Menschengeschlechtes. Dagegen deutet eine Auffassung von Menschenrassen als unterschiedliche Arten auf eine polygenetische Annahme über die Herkunft des Menschen hin.

38 Er setzte dabei in seiner Einteilung einen runden Schädel in die Mitte, den berühmten Schädel einer "Georgianerin" und nannte diese Varietät die kaukasische. In den breiten beziehungsweise schmalen Schädelformen der aus Asien beziehungsweise Afrika stammenden Schädel seiner Sammlung sah er die größten Abweichungen von der kreisförmigen Mitte und nahm dazu noch zwei Übergangsstufen an, die Malayen sowie die Indianer Amerikas. Vgl. Blumenbach (1796: o.S.), wo er kurz die "physiognomischen Unterscheidungszeichen dieser 5 Rassen" skizziert, bevor die Porträts der "Characterischen Musterköpfe von Männern aus den fünf Hauptrassen im Menschengeschlechte» folgen.

39 Der Leipziger Geograph und Publizist Oscar Peschel führt es im Kapitel "Anthropologie" seiner Geschichte der Erdkunde bis auf A. v. Humboldt und Carl Ritter, München 1865: 678-681, hier S. 680f., als besonderen Verdienst Blumenbachs an, neben den fünf Hauptrassen auch auf die vielzähligen Übergangsformen hingewiesen zu haben. Vgl. Wilhelm E. Mühlmanns Bewertung in seiner Geschichte der Anthropologie, Wiesbaden 1984: $58 \mathrm{f}$

In neuerer Zeit kommt dem Göttinger Medizinhistoriker Frank W. P. Dougherty das besondere Verdienst zu, Blumenbachs Werk wieder ins rechte aufklärerische Licht gerückt zu haben. Neben der Konstruktion der fünf Hauptrassen, "Variationen, die die menschliche Körperform annehmen könne oder vielmehr historisch angenommen habe" müsse, so Dougherty (1990: 108), berücksichtigt werden, daß für Blumenbach, "die Schattierungen, die der individuelle Ausdruck eines jeden Merkmals in der Natur zeige, [die] in der Tat ins Unendliche nuancieren und die scharf gezeichneten Grenzen zwischen den Idealtypen verwischen". Dougherty (1990: 108).

Mit Blick auf diese Übergänge hatte sich auch Herder ([1785] 1967: 258) im siebten Buch seiner Ideen gegen eine starre Klassifikation von Menschenrassen gewandt: "Die Farben verlieren sich ineinander [...]; und im Ganzen wird zuletzt alles nur Schattierung eines und desselben großen Gemäldes, das sich durch alle Räume und Zeiten der Erde verbreitet". 


\section{EINTEILUNGEN}

Wenngleich wir Heutigen auch um die Unmöglichkeit des Versuches wissen, das Kontinuum unendlich vieler menschlicher Erscheinungsformen empirisch zu erfassen und zu zerteilen, sahen im 19. Jahrhundert viele Wissenschaftler des deutschen Sprachraumes gerade hierin das vornehmliche Arbeitsfeld der physischen Anthropologie. Anders als noch Blumenbach wollten sie sich nicht damit zufriedengeben, daß Menschenrassen als Varietäten, als Spielarten des Menschengeschlechtes, "durch so mancherlei Abstufungen und Übergänge so zusammenfließen, daß sich daher auch keine andere[n] als sehr willkürliche Grenzen zwischen ihnen festsetzen lassen ${ }^{40}$.

So strebte etwa Virchow als langjähriger spiritus rector der physischen Anthropologie danach, "die allgemeinen Züge in der körperlichen Erscheinung der einzelnen Stämme zu erkennen und die Merkmale festzustellen, an welchen sich einerseits die Zusammengehörigkeit der einzelnen Glieder eines Stammes im Gegensatze zu benachbarten Stämmen, andrerseits aber auch die Verwandtschaft verschiedener Stämme unter einander erkennen lässt" ${ }^{41}$. Der menschliche Körper und besonders der Schädel wurde dabei in einer Vielzahl zu messenden Dimensionen geradezu atomisiert, und es wurde wie manisch versucht, Verschiedenheiten dingfest zu machen - indes blieben die Resultate fragwürdig.

Parallel zur Erhebung körperlicher Meßdaten erfolgte die photographische Dokumentation der körperlichen Erscheinungsform des Menschen als Hauptgegenstand einer wissenschaftlichen Anwendung der Photographie, denn Photographien konnten, so Virchow, "die Beschreibung auf das Glücklichste ergänzen" ${ }^{42}$. Einzig mit dem neuen Medium der Photographie schien es den Zeitgenossen möglich, die oben angesprochenen Übergangsformen in genügender Anzahl, Schnelligkeit und Präzision zu dokumentieren.

Adolf Bastian (1826-1905), Mitbegründer der Berliner Gesellschaft für Anthropologie, Ethnologie und Urgeschichte, Gründungsdirektor des Berliner Museums für Völkerkunde und auf dem Felde der Ethnologie ebenso einflußreich wie der bereits erwähnte Virchow für die physische Anthropologie, plädierte ebenfalls für einen umfänglichen wissenschaftlichen Ge-

40 So Blumenbach (1814: 101) in seinem Handbuch der Naturgeschichte, zit. n. Bitterli (1982: 347).

41 Virchow (1875: 590). Hervorhebung Virchow.

42 Virchow (1875: 584). 
brauch der Photographie. Er wies mit Nachdruck darauf hin, daß ues für sichere Fundamentirung der neu entstehenden Wissenschaft der Ethnologie unumgängliche Vorbedingung sein würde, Reihen photographischer Darstellungen aus den verschiedenen Menschenraçen in grösserer Zahl zu erhalten und zu sammeln" ${ }^{43}$.

\section{TYPENLEHREN}

Der Begriff typos ${ }^{44}$ vom griechische typtein ${ }^{45}$, für schlagen, hauen, bedeutet wörtlich "Schlag, das durch einen Schlag hervorgebrachte, die Spur eines Eindrucks" ${ }^{46}$. Im 16. Jahrhundert über das lateinische typus in den deutschen Sprachgebrauch gelangt, trug das Wort neben der theologischen Bedeutung als Vorbild ${ }^{47}$ zugleich die Bedeutung "Merckmahl, Fußtapfen" ${ }^{48}$, worin auf eine Ur- oder Stammform gedeutet wurde, die allen Angehörigen einer Gruppe, gewissermaßen als Abformung davon, zu eigen wäre.

Dem antiken Ursprung des Begriffes folgend, wurden "Einzelnwesen in ihrer sinnlichen Erscheinung als die Abbilder von Urbildern betrach-

43 B.[astian] (1872: 392).

44 Krech hat 1984 das Aufkommen des Typus-Begriffs in der französischen physischen Anthropologie thematisiert, widmet der Herkunft und dem wissenschaftlichen Kontext des Typus'-Begriffes allerdings nur wenig Aufmerksamkeit. Edwards (1990: 240f.) hat ihrem Aufsatz vorzugsweise die angelsächsische Verwendung des Terminus' im Blick.

45 Grebe (1963: 726).

46 Schulz (1981: 546). Der Begriff bedeutet darüber hinaus noch Gepräge, Form, Gestalt, Abbild, Vorbild, Urbild, Muster, Modell, Charakter.

47 Zedler (50. [1746] 1982: 751). Vgl. Weigand (2. [1910] 1968: 1093) und besonders Schulz (5. 1981: 546-554). Wie es 1746 weiter in Johann Heinrich Zedlers Grossem Vollständigen Universal-Lexikon hieß, wurde typus verstanden als "Zeichen [...], welches etwas zukünfftiges und vortrefflichers andeutet, davon GOtt selbst die Abbildung giebet." Zedler (50. [1746] 1982: 753). Damit waren Personen oder Geschehnisse aus dem Alten Testament gemeint, die auf das Neue Testament vorausweisen sollten, in denen sich also "der Sohn Gottes [...] vor seiner Menschwerdung [...] geoffenbaret", ebd., hatte. "[...]; also ist Typus auch in der Heil. Schrifft ein Exempel, dem man nachfolgen soll." Zedler (50. [1746] 1982: 751). Die Typologie als Lehre von den Typen hatte, ähnlich wie der Typus-Begriff, zunächst nur eine theologische Bedeutung als "Wissenschaft von der vorbildlichen Beziehung", Brockhaus (11. 1836: 450), zwischen Personen und Geschehnissen des Alten und des Neuen Testaments. Der Lexikoneintrag sah allerdings in der "typischen Exegese", Brockhaus (11. 1836: 450), nur "phantasiereiche Combinationen", welche "in der Regel nur wenig Gehalt für die strenge Wissenschaft darbieten" Brockhaus (11. 1836: 450).

${ }_{48}$ Zedler (50. [1746] 1982: 751). 
tet ${ }^{49}$. Umgekehrt sollte aus der Vielzahl der sinnlich wahrnehmbaren Erscheinungen ein gedachtes Urbild wiedererkannt werden können und sich der Typus aus der vergleichenden Zusammenschau vieler Angehöriger einer bestimmten Gruppierung ergeben. In diesem Sinne einer allen Individuen gemeinsamen ideellen Grundform, der "Gesammtvorstellung einer Sache, ihrem bleibenden und wesentlichen Merkmale nach" ${ }^{50}$ hatte der Begriff des Typus' als systematische Kategorie seit dem 18. Jahrhundert ${ }^{51}$ in den Naturwissenschaften Eingang gefunden.

Für die Ethnologie suchte sich Bastian 1869 den Typus-Begriff in einem Text, "Das natürliche System in der Ethnologie" ${ }^{52}$, zunutze zu machen. Er bezog sich dort unter anderem auf die Typenlehren des Biologen Georges de Cuvier (1769-1832) sowie des Naturforschers und Anthropologen Karl Ernst von Baer (1792-1876) ${ }^{53}$ und wies daneben auf Systematisierungskonzepte hin, wie sie um die Jahrhundertmitte für die Ordnung chemischer Verbindungen ${ }^{54}$ entwickelt worden waren. In diesem

49 Brockhaus (11. 1836: 450).

50 Brockhaus (11. 1836: 450). Weiter heißt ist dort die Rede etwa vom "Typus einer Thiergattung, [...], eines Mythus, der sich etwa mannigfaltig modificirt bei verschiedenen Völkern findet, als der allen diesen Modificationen gemeinschaftlichen Grundzüge.. Ebd..

51 Vgl. Schulz (5. 1981: 546) und ebenso Grimm (11. I, 2. 1952: 1961).

52 Der Text erschien auf den ersten Seiten der von ihm und anderen 1869 ins Leben gerufenen Zeitschrift für Ethnologie und trug gleichsam programmatischen Charakter. Der Begriff des "natürlichen Systems" ist als Entlehnung aus der botanischen Systematik zu verstehen. Dort wurden und werden sogenannte künstliche von natürlichen Systemen zur Klassifikation unterschieden. Ein künstliches System ordnet die Pflanzen nach Übereinstimmungen oder Verschiedenheiten eines bestimmten äußerlichen Merkmales. So werden im Klassifikationsschema des schwedischen Arztes, Naturforschers und Botanikers Carl von Linné die verschiedenen Bestandteile des pflanzlichen Geschlechtssystems als Schlüsselmerkmale zur Einteilung verwendet. Unter einem natürlichen System, wie zuerst von dem französischen Arzt und Botaniker Bernard de Jussieu entworfen, werden dagegen Klassifikationsschemata verstanden, in denen sich die einzelnen Arten aufgrund von typischen Ähnlichkeiten und Verwandtschaften in ihrem ganzen Erscheinungsbild anordnen lassen.

53 Der durch seine Katastrophen- oder Kataklysmen-Theorie hervorgetretene Cuvier vertrat in einer Typenlehre die Auffassung, daß sich das Tierreich in vier verschiedene Typen einteilen ließe: Wirbeltiere, Gliedertiere, Weichtiere und Strahltiere. Jede dieser Formengruppen hätte eine eigene Entwicklung durchlaufen, ein Zusammenhang wäre höchstens am Ursprung der zugrundeliegenden Typen denkbar. Später sollte Baer diese Einteilung aufgreifen.

5i Vgl. Bastian (1869: 9f.). Bastian bezog sich dort explizit auf die Typenlehren der Chemiker Jean Baptiste Dumas', Carl Friedrich (Charles) Gerhardts gemeinsam mit Auguste Laurent und erwähnt insbesondere diejenige Friedrich August Kekulés (von Stradonitz). 
Zusammenhang betrachtete Bastian verschiedene Typen "nicht etwa [als] durch verschiedene Constitution scharf getrennte Klassen von Verbindungen, es sind vielmehr bewegliche Gruppen, in welche man immer die Verbindungen zusammenstellt, welche den Eigenschaften nach, die man besonders hervorheben will, eine gewisse Analogie zeigen" 55 .

Der Typus-Begriff beinhaltete die allen Angehörigen einer "Art oder Gattung gemeinsame (ideelle) Grundform" ${ }^{56}$. Wesentlich ist dabei der Begriff "ideell", denn ein solcher Typus war stets nur eine gedachte wesenhafte Grundform der Art, eine Idee, die Essenz, nicht aber ein wirklich existierender Organismus ${ }^{57}$. Ein "Realrepräsentant ${ }^{58}$ konnte den angenommenen Typus nicht wirklich verkörpern, sondern sich ihm lediglich annähern. Die unterschiedlichen Variationen ${ }^{59}$ stellten mehr oder weniger gelungenene Realisierungen ${ }^{60}$ der zugrundeliegenden Essenz oder Idee dar, wie etwa Johann Wolfgang von Goethes (1749-1832) "Urpflanze" ${ }^{61}$. Folgt man der Darstellung des Biologen Ernst Mayr, dann war noch zu Zeiten

55 Bastian (1869: 10). Er griff damit bereits auf das heutige Verständnis der Typenlehren, beziehungsweise wie es im modernen Sprachgebrauch heißt, der Typologien, voraus. Danach wird ein Typus, ausgehend von der Bedeutung Vorbild, Urbild, Urform, verstanden als "die unveränderliche, allgemeine Grundform, Grundgestalt, die ähnlichen bzw. vergleichbaren Individuen [...] zugrundeliegt; [eine] in charakteristischen Wesenszügen bei allen Vertretern der gleichen Art, Klasse signifikant ausgeprägte Grundform". Schulz (5. 1981: 546).

56 Meyers Konversations-Lexikon (15. 1878: 235).

57 Vgl. dazu Adolf Meyer-Abichs Einleitung, "Goethes Naturforschung in ihrer geistesgeschichtlicher Gestaltw, zu seiner Biologie der Goethezeit, (1949: 27-34), in der er nachdrücklich auf die antiken Wurzeln dieser Vorstellung hinweist. Das Verständnis des Typus-Begriffes als die allen Gruppenmitgliedern analoge Grundform wies so auch auf den Sprachgebrauch Johann Wolfgang von Goethes zurück, der in seinen Schriften Zur Morphologie des Jahres 1820 nachdrücklich die gedachte, ideelle Bedeutung des Typus-Begriffes als Abstraktion von den real gegebenen Individuen zum Ausdruck gebracht hatte: "Schon aus der allgemeinen Idee eines Typus folgt, daß kein einzelnes Tier als ein solcher Vergleichungskanon aufgestellt werden könne; kein Einzelnes kann Muster des Ganzen sein." (1969: 141).

58 Meyer-Abich (1949: 33).

59 Goethe verstand in seiner idealistischen Morphologie die tatsächlich existierenden Organismen, die stufenweise durch Übergänge verbundenen Abwandlungen eines Typus als Metamorphosen.

${ }^{60}$ Ernst Mayr (1990: 50) spitzt dies zu, wenn er schreibt: "Für den Essentialisten [...] war das allein Reale die Idee und die Variation schlicht ein "Fehler"."

${ }^{61}$ Goethes "Urpflanze" war keineswegs ein tatsächlich existenter Vorfahre der rezenten Pflanzenwelt, sondern im Sinne des typologischen Denkens ein ideelles Modell, ein "Muster aller Pflanzen". Meyer-Abich (1949: 31f.). 
Charles Darwins (1809-1882) diese "essentialistische" Denkweise in der deutschsprachigen Biologie vorherrschend. Arten wurden danach verstanden aals durch konstante Merkmale charakterisierte und durch scharfe, in keiner Weise überbrückbare Diskontinuitäten voneinander getrennte Klassen" ${ }^{62}$. Zu diesem Verständnis der Arten als konstanter, nicht wandelbarer Typen gehörte ein Ordnungsprinzip, das die Organismen nach der Nähe oder Ferne zu einem ideellen Typus in eine kontinuierliche räumliche Anordnung brachte. Da alle Lebewesen durch die göttliche Schöpfung zur gleichen Zeit erschienen wären und keine neuen hinzukommen könnten, wurden sie auch als unendliche Kette aller Lebewesen nebeneinander gestellt.

Die Vorstellungen eines "musée photographique des races humaines" knüpften an jene "essentialistische" Denkweise an. Indem die Rassentypen und ihre zahllosen Übergangsformen ebenfalls geographisch angeordnet, nebeneinander stehen sollten, sähe man Bastian zufolge "Von Bild zu Bild den Uebergang von einer Race zur andern for [t]schreiten" " ${ }^{63}$.

\section{"TYPISCHE CHARAKTERE"}

Zwar vertrat auch Bastian die Auffassung, daß nur mit Hilfe der Photographie "die typischen Charaktere der einzelnen Völker treu dargestellt, die wesentlichen Eigenschaften der Racen unverfälscht wiedergegeben werden können" ${ }^{64}$. Für ihn ergäbe sich jedoch erst in einer Art geistiger Zusammenschau, "das richtige Bild einer Race, eines Volkes [...] nur aus einer größern Anzahl von Bildern, die zusammengehalten sich gegenseitig ergänzen und ein Gesammtbild erzeugen, das allerdings ideal, aber doch wahr ist" ${ }^{65}$. In einer solchen Zusammenschau oder auch "descriptiven Beschreibung der Menschengeschlechter auf Erden" ${ }^{66}$ bestand für Bastian der erste Arbeitsschritt seiner Wissenschaft vom Menschen. Wesentlich an Bastians Vorstellung eines "Gesammtbildes" ist dabei der Begriff "ideal", denn ein solcher Typus war, wie oben gezeigt, stets nur eine gedachte und nicht in der Wirklichkeit existierende Form, die sich aus der vergleichenden Zusammenschau vieler Angehöriger einer bestimmten Gruppierung gedanklich ergeben sollte. Indes stieß solch ein imaginatives Verfahren zur Bil-

\footnotetext{
62 Mayr (1990: 50).

63 Dr. B.[astian] (1873: 11).

${ }_{64}$ Dr. B.[astian] (1873: 10f.). Hervorhebungen Bastian.

65 Dr. B.[astian] (1873: 12).

to Bastian (1875: 136).
} 
dung ideeller Typen auch auf berechtigte $\mathrm{Kritik}^{67}$ - so etwa in der 1874 erstmals erschienenen Völkerkunde des Leipziger Geographen und Publizisten Oscar Peschel (1826-1875). Peschel betrachtete die gedachte, subjektive Zusammenschau der Erscheinungen zu einem Idealtypus ohne zugrundeliegende Meßergebnisse als eine gefährliche Täuschung. Hierdurch würde nicht ein, im empirischen Sinne repräsentativer Vertreter ermittelt. Vielmehr würde die Subjektivität und die von Vorurteilen geprägte Erwartungshaltung des Reisenden ${ }^{68}$ dazu führen, daß "als Vertreter eines Typus unter sehr Vielen derjenige herausgesucht wird, welcher am schärfsten sich von den Gliedern anderer Menschenstämme absondert" ${ }^{69}$. So würde, etwa in Bastians geistiger Zusammenschau, "die Auswahl von Typen nach dem Augenmasse zu künstlerischer Willkür verleiden [sic!], ${ }^{70}$. Peschel setzte dagegen eine empirisch fundierte Vorgehensweise, wonach der "Vergleich der gemessenen Ziffern untereinander [...] das einzige Verfahren ist, welches bislang der Wissenschaft zu Gebote stand ${ }^{71}$.

\section{"MITTLERE ZAHLENWERTHE"}

Vogt hatte in ähnlicher Weise wie auch Bastian den Standpunkt vertreten, "daß die Photographie das leichteste und sicherste Mittel bietet, Rassenbilder massenhaft zu verfertigen" ${ }^{72}$. Darüber hinaus zeigen sich doch unterschiedliche Positionen wie dem Anspruch der Wissenschaft genügt werden könne, "welche nicht einzelne, vielleicht charakteristische Gesichter, sondern viele Gesichter verlangt, aus welchen sie den Mitteltypus herauszufinden im Stande sei, ${ }^{73}$. Vogts Weg zu einer solchen Typisierung ließe sich im Gegensatz zu Bastians gedachtem, ideellem Typus mit dem Begriff eines errechneten Durchschnittstypus' umreißen. Wenngleich Vogt auch keine präzisen Angaben über die Verwendung der Photographie in diesem Sinne machte, wird doch an anderer Stelle seiner Vorlesungen das Ziel solch massenhafter Bildproduktion und -konsumtion deutlich: Anstel-

\footnotetext{
67 Auch Sir Francis Galton lehnte dieses ideelle Verfahren ab (1878: 97). Seiner Ansicht nach kämen auch nicht zwei der größten Künstler zu einer übereinstimmenden Darstellung von der Physiognomie eines Menschen.

68 Vgl. Peschel (1875: 15).

69) Peschel (1875: 15).

70 Peschel (1875: 63).

71 Peschel (1875: 63).

72 Vogt (1. 1863: 85).

73 Vogt (1. 1863: 85).
} 
le singulärer und mit subjektiven Fehleinschätzungen behafteter Beschreibungen, forderte er empirisch gesicherte Aussagen. Hierfür sei es nötig, Beobachtungen und Vermessungen so häufig durchzuführen, "daß aus der Masse der erhaltenen Resultate eine Mittelzahl gewonnen werden kann, welche das Gesetz darstellt, um dessen Norm herum die einzelnen Resultate schwanken" ${ }^{74}$. Vogt bezog sich hierin auf den belgischen Mathematiker und Astronomen Lambert Adolphe Jacques Quételet (17961874) ${ }^{75}$, demzufolge sich meßbare organische Phänomene wie zum Beispiel die Körpergröße in einer Population, im Sinne einer Gausschen Normalverteilung gleichmäßig um ihren Mittelwert, den sogenannten Durchschnittstypus gruppierten.

Im September des Jahres 1861 fand auf Initiative des Göttinger Naturforschers und Physiologen Rudolph Wagner (1805-1864) und seines St. Petersburger Kollegen Baer eine Zusammenkunft verschiedener Anthropologen in Göttingen statt, um ein einheitliches Vorgehen bei Vermessung und Bilddarstellung zu vereinbaren. Baer und Wagner erschien es wünschenswert, mit Blick auf den wissenschaftlichen Hauptstreitpunkt ihrer Zeit ${ }^{76}$, die Auseinandersetzungen um eine monogenetische oder polygenetische Abstammung des Menschengeschlechtes, "dass man von den jetzt lebenden Völkern und und ihren Hauptverzweigungen durch Vergleichung vieler Individuen die Mittelformen und das Verhältniss ihrer Abweichungen zu bestimmen suche, sei es durch Maasse oder bildliche Darstellung" ${ }^{77}$. Auch bei Baer und Wagner führte der Weg zur Bestimmung solch "typischer Formen" über eine möglichst große Anzahl von Einzelmessungen und die Berechnung der aaus einzelnen Messungen gezogenen Mittel und das Verhältnis dieser Mittelmaasse gegen einander ${ }^{78}$ Allerdings seien bislang solcherart durchgeführte Forschungen noch "sehr dürftig [...] und man wird mit Ungeduld die anthropologischen Studien der Gebrüder Schlagintweit und der Novara-Expedition erwarten" ${ }^{79}$.

74 Vogt (1. 1863: 25).

75 Quételets Arbeit Sur l'homme et le developpement de ses facultés, ou Essai de physique sociale erschien im französischen Original erstmals 1835. Die deutsche Ausgabe folgte unter dem Titel: Ueber den Menschen und die Entwicklung seiner Fähigkeiten oder Versuch einer Physik der Gesellschaft. Stuttgart 1838. Vogt (1863: 27) erwähnt Quételet in seiner zweiten Vorlesung über anthropologische Meßmethoden.

76 Vgl. Baer/Wagner (1861: 8, 17-24, 68).

77 Baer/Wagner (1861: 14).

78 Baer/Wagner (1861: 11).

79 Baer/Wagner (1861: 11). Hervorhebung Baer/Wagner. Gemeint sind die Expeditionsresultate von der Reise der bereits oben erwähnten Brüder Schlagintweit nach Vorderindien und Hochasien in den Jahren 1854-1857. 


\section{“KEINE DURCHSCHNITTSMENSCHEN"}

Noch während der Weltumsegelung der österreichischen Fregatte "Novara" (1857-1859) hatten der Reisende und Publizist Karl Ritter von Scherzer (1821-1903) und der Schiffsarzt und Botaniker Eduard Schwarz (1831-1862) erstmals ein einheitliches anthropometrisches Meßschema ${ }^{80}$ vorgestellt, das die bis dahin bestehende Unsicherheit über die zu nehmenden Maße sowie deren mangelhafte Vergleichbarkeit überwinden wollte. Aus möglichst vielen Daten von Körpermessungen sollten, im Sinne statistischer Mittelwerte, sogenannte Durchschnittstypen abgeleitet werden, um eine Einteilung von Menschenrassen auf empirisch gesicherter Grundlage vorzunehmen.

Scherzer und Schwarz erdachten weiterhin, aus der Einsicht in die unzureichende Aussagekraft und Gültigkeit einzelner Abbildungen, ein zeichnerisches Dokumentationsverfahren, in dem das durchschnittliche Gesichtsprofil einer Population in einem Koordinatensystem dargestellt werden konnte ${ }^{81}$. Schemazeichnungen verschiedener Populationen sollten zudem noch direkte Vergleichsmöglichkeiten bieten, indem sie, auf Transparentpapier gezeichnet, übereinander gelegt werden konnten.

Ein ähnliches Verfahren des Übereinanderlegens verschiedener Abbildungen stellte dann Sir Francis Galton (1822-1911) erstmals 1878 mit den sogenannten "composite portraits" vor, zu deutsch "Mittelbildern" ${ }^{82}$. Hierbei wurden photographische Negative von Porträtaufnahmen einer bestimmten Population übereinander gelegt, um tatsächlich ein photographisches Durchschnittsbild jener Gruppe zu erzielen. Unter den deutschen Anthropologen waren die verschwommenen Ergebnisse allerdings umstritten. Die "Mittelbilder" hatten, so etwa für Fritsch, "durch das Auslöschen aller charakteristischen Züge etwas Leeres, Unbefriedigendes, das unserem Streben nach Erkenntnis der Komponenten eines Rassenbildes nicht genügt" ${ }^{83}$. Das Verfahren Galtons konnte sich nicht durchsetzen. Auch Scherzers und Schwarz' Schemazeichnungen sollten nicht bloß wegen der Kompliziertheit ihres Konstruktionsverfahrens eine nur kurzzeitig beachtete Kuriosität bleiben.

80 Vgl. Scherzer/Schwarz (1859).

81 Vgl. Scherzer/Schwarz (1859: 20-23).

82 Galton legte sein Referat, "Composite Portraits made by combining those of many different persons into a single resultant figure", dem Londoner Anthropologischen Institut am 30. Mai 1878 vor. Es wurde veröffentlicht in Nature. 18. 1878: 97-100. Der Text erschien unter dem Titel "Composite Portraits" ebenfalls im Journal of the Anthropological Institute. 8. 1879: 132-144.

83 Fritsch (1911: 22). Vgl. Stenger (1938: 88). 
Zum Scheitern der Mittelwertbilder trugen zum einen ihre technischen Unzulänglichkeiten bei. Dahinter verbarg sich zum anderendie Ablehnung der empirisch gewonnenen Durchschnitts- oder Mittelwerttypen und damit die Vorherrschaft einer ideellen Typenbildung in der Goetheschen Traditionslinie, wie sie auch von Bastian vertreten wurde. Er erklärte 1873 in einer Rezension kategorisch: "Es giebt keine Durchschnittsmenschen, und wenn sie künstlich construirt werden, wie vielfach versucht wurde, so entstehen mehr oder weniger Fratzen, welche die individuelle Anschauung des Bildners, aber nicht die Wirklichkeit darstellen" ${ }^{84}$.

\section{"VERGLEICHUNGEN"}

Die Vorstellung eines "musée photographique des races humaines" mit seinen beabsichtigten Tableaus der als typisch angesehenen Vertreter verschiedener Rassen und Regionen ist als leitender Gedanke in den Überlegungen zur wissenschaftlichen Nutzung der Photographie im 19. Jahrhundert ${ }^{85}$ anzusehen. Wie auch in zahlreichen Gründungen ethnologischer Museen im letzten Jahrhundertdrittel kam darin der Gedanke zum Ausdruck, dem befürchteten "Aussterben der Naturvölker" zuvorzukommen und wenigstens einen musealen Beleg als memento mori für die Forschung zu bewahren: Was für die Photographie von Beginn an mit Blick auf eine Dokumentation von Hieroglyphen und Architekturdenkmälern vorgeschlagen wurde, galt in der Völkerkunde entsprechend für vermeintlich ursprünglich oder isoliert lebende Ethnien, für «[...] all die mehr oder weniger der Zerstörung entgegengehenden Reste jener zum Theil schon entschwun-

84 Dr. B.[astian] (1873: 12). Hervorhebungen Bastian. Wenngleich auch im Sprachduktus der Veröffentlichungen Bastians immer wieder mathematisch-naturwissenschaftliche Begriffe wie "Gedankenstatistik", "Durchschnitt", "logisches Rechnen" etc. auftauchen, blieb er doch weit davon entfernt, diese auch tatsächlich in empirischem Sinne zu verwenden. Vgl. Fiedermutz-Laun (1970: 38) und Köpping (1983: 32f.).

85 Vgl. Falconer (1984/86: 25-30). Wenngleich auch die oben erwähnten Pläne Serres' für ein "photographisches Museum der Menschenrassen" in ihrem Universalitätsanspruch weitgehend Makulatur blieben, so sind dennoch im deutschen Sprachraum einige regionale Realisierungsversuche zu verzeichnen: Fritsch, Gustav: Die Eingeborenen Süd-Afrikas. Atlas enthaltend 30 Tafeln Raçentypen. Breslau 1872. Falkenstein, Julius: Afrikanisches Album. Die Loango-Küste in 72 Original-Photographien (35 Blatt) nebst erläuterndem Texte. Berlin 1876. Buchta, Richard: Die oberen Nil-Länder. Volkstypen und Landschaften. Dargestellt in 160 Photographien. [...J. Berlin 1881. Friederichsen, Ludwig (Hg.): Süd-See Typen. Anthropologisches Album des Museum Godeffroy in Hamburg. Hamburg 1881. 
denen, zum Theil vor der Sonne der europäischen Cultur vergehenden Naturvölker [...]" ${ }^{86}$.

Die Photographie sollte Menschen aus den verschiedensten Regionen der Erde nebeneinanderstellen, die räumliche und zeitliche Beschränkung der einzelnen Forschungsreisen aufheben und an die Stelle cler Ungleichzeitigkeit der einzelnen Aufnahmen die Gleichzeitigkeit eines Talbleaus, eines Albums, eines photographischen Museums setzen. Für Vogt gestattete die Photographie, sonst durch Zeit und Raum getrennte Gegenstände unmittelbar mit einander zu vergleichen. [...]. Ohne die Photographie gibt nur subjective Auffassung in Zeichnung oder Erinnerung uns das Mittel der Vergleichung. Meist können wir die Rassen nicht neben einander stellen. [...]. Kann die Photographie freilich nicht die verschiedenen Rassen zur Stelle schaffen, so kann sie doch einigermaßen den Mangel durch ihre naturgetreue Nachbildung ersetzen ${ }^{87}$.

\section{Types Prinçipaux Des DifféREnTes RaÇES Humaines}

Baer und Wagner schien anläßlich der Göttinger Zusammenkunft im Jahre 1861 eine Lösung des Problems, "ob die Verschiedenheiten im Baue der menschlichen Körper als ursprünglich oder als geworden zu betrachten sind" ${ }^{88}$, nur auf Grund ausreichenden empirischen Materials ${ }^{89}$ möglich. In diesem Sinne forderten sie die Anlage "einer Sammlung, in welcher fast alle Völker des Erdbodens mit ihren Variationen repräsentirt sind, oder eine Sammlung gut ausgeführter zuverlässiger Abbildungen der typischen Formen oder wenigstens übereinstimmende Messungen, die nach demselben Principe durchgeführt sind" ${ }^{90}$. Baer sollte aus diesem Verständnis heraus in den 1860er Jahren ein photographisches Tafelwerk publizieren, die Types Prinçipaux Des Différentes Raçes Humaines dans les cinq parties Du Monde ${ }^{91}$.

86 So etwa der Hamburger Arzt und Anthropologe Rudolf Krause (1881: 580) im anthropologischen Teil des Sammlungsinventars des Museum Godeffroy in Hamburg.

87 Vogt (1863: 85f.).

$88 \quad$ Baer/Wagner (1861: 11).

89 Vgl. ebd. (1861: 9, 11, 14, 16, 17, 41f., 68).

90 Baer/Wagner (1861: 11).

91 Baer, Karl Ernst von: Types Prinçipaux Des Différentes Raçes Humaines dans les cinq parties Du Monde, modélés sous la direction du Pr. Baer de St. Petersbourg. Ohne Ort und Jahr. 
Als Vorlage für die Photographien dienten Reihen von Halbreliefs aus Steinpappe ${ }^{92}$. Sie zeigten "plastische Darstellungen der verschiedenen National-Typen des Erdballs" ${ }^{93}$ auf insgesamt sieben Tafeln. Später erschienen fünf Supplementtafeln mit Photographien von Gips- oder Wachsbüsten der männlichen Vertreter der fünf Hauptvarietäten, angelehnt an das oben erwähnte Einteilungsmodell Blumenbachs. Vor dem Hintergrund der vielfältigen und kontrovers diskutierten Schemata zur Einteilung von Menschenrassen war Baer allerdings "entschieden dagegen, diese Typen nach gewissen Hauptstämmen und Verzweigungen zu ordnen, weil die Variationen des Menschengeschlechtes so mannigfaltig sind und in einander übergehen, daß es immer etwas Willkürliches bleibt, wieviel Hauptformen man annehmen will ${ }^{94}$. Aus Mangel an sicherer Kenntnis über die Abstammung des Menschen —us läßt sich nämlich nicht bestimmen, welche Variante man als aus einander entstanden betrachten soll ${ }^{95}$ - verzichtete Baer zugleich auf eine systematische Anordnung der Abbildungen. Unter diesen Umständen schien es ihm am zweckmäßigsten, "die Typen der verschiedenen Völker geographisch zu vertheilen, also, wie sie in Wirklichkeit bestehen, ohne alle willkürliche Annahme über ihre Abstammung ${ }^{96}$.

Baers Präsentationsschema der verschiedenen Daseinsformen des Menschen behielt, unter Zuhilfenahme der Photographie, das überlieferte Schema einer räumlichen Anordnung der Lebewesen in der Naturgeschichte bei. Würde man die Reihen nebeneinanderstehender Einzelporträts nicht, wie auf den Tafeln, untereinander anordnen, sondern immer weiter nebeneinander fortfahren, so ergäbe sich ein langes Band, auf dem die "Kette der Wesen" erschiene.

92 Ein Bildhauer namens Heuser fertigte um 1861 für Baer fünf Tafeln mit je 16 solcher Halbrelieffiguren an. Steinpappe ist eine "Masse aus aufgeweichtem Papier", die "mit Thon, Kreide und Leimwasser versetzt" wurde. Kürschner, Joseph (hg.): Pierers Konversationslexikon. Stuttgart 11. 18927: 1384. Hierüber informiert ein Text Baers aus dem Jahre 1861: Typen von National-Physiognomien aus Steinpappe.

Anschließend wurden die Halbrelieffiguren photographiert und auf fünf Tafeln ohne Angabe von Ort und Erscheingsjahr publiziert. Die später folgenden Supplementtafeln, wiederum zwei mit Photographien von Halbrelieffiguren und fünf mit Photographien von Gips- oder Wachsbüsten, blieben ebenfalls undatiert. Auf allen Tafeln findet sich der Hinweis auf eine Prämiierung bei der dritten Weltausstellung in London im Jahre 1862. Dabei bleibt unklar, ob die Arbeit Heusers präsentiert und ausgezeichnet wurde oder die photographische Wiedergabe.

93 Baer (1861: 1).

94 Baer (1861: 2).

95 Baer (1861: 3).

\% Baer (1861: 3). 


\section{ANTHROPOLOGISCH-ETHNOLOGISCHES ALBUM IN PHOTOGRAPHIEN}

Bereits anläßlich der Gründung der Zeitschrift für Ethnologie durch Bastian und andere im Jahre 1869 war der Wunsch laut geworden, "in grösserem Maassstab eine Sammlung photographischer Rassenportrait's anzulegen, die unumgänglich gefordert wird, um den ethnologischen Untersuchungen die sichere Basis thatsächlicher Anschauung zu geben" ${ }^{97}$. Bastian hatte wesentlich Anteil daran, daß die Berliner Gesellschaft für Anthropologie, Ethnologie und Urgeschichte, eine der bedeutendsten wissenschaftlichen Gesellschaften dieses Fachgebietes im deutschen Sprachraum, vom Zeitpunkt ihrer Gründung im Jahre 1869 an, eine photographische Sammlung ${ }^{98}$ einrichtete. Innerhalb kurzer Zeit nahm sie einen beträchtlichen Umfang an, so daß Bastian 1872 einen "Mittelpunkt zur Vereinigung des weit Zerstreuten und eines Organes der Veröffentlichung, 99 forderte, "um die Zugänglichkeit für weitere Kreise zu ermöglichen" ${ }^{100}$. Eine solche Möglichkeit, gleichsam den alten Wunsch Herders und Blumenbachs zu erfüllen und das Bildmaterial der wissenschaftlichen Öffentlichkeit zur Verfügung zu stellen, sollte sich durch die Publikation des $A n$ thropologisch-Ethnologischen Albums in Photographien [...] $]^{101}$ ergeben. Dammann als Herausgeber stellte damit in den Jahren 1873 und 1874 ein großformatiges Mappenwerk vor, das in Gestalt von 50 Tafeln mit 642 Photographien, geographisch geordnet, eine Vielzahl ethnischer Gruppen aus allen Erdteilen präsentierte. Mit Dammanns Album hielt die Photographie Einzug als Illustrationsmedium in die allgemeine und vergleichende deutschsprachige Literatur der Disziplinen physische Anthropologie und Ethnologie.

97 [Bastian] (1869: 400).

98 Ebd.. Anläßlich der Trauerfeier zu Ehren des am 3. Februar 1905 in Port of Spain auf der Karibikinsel Trinidad verstorbenen Bastian wies der damalige Vorsitzende der Berliner Gesellschaft für Anthropologie, Ethnologie und Urgeschichte, der Anthropologe Abraham Lissauer, auf Bastians besondere Verdienste bei der Begründung der Zeitschrift für Etbnologie und bei der Anlage der Photographiensammlung der Berliner Gesellschaft hin: So "regte Bastian schon damals [im Gründungsjahr der Berliner Gesellschaft, 1869] die Sammlung von anthropologischen und ethnographischen Photographien an, welche sich im Laufe der Jahre zu einem kostbaren Besitz unserer Gesellschaft entwickelt hat." Lissauer (1905: 235).

99 Ebd.

100 Ebd.

101 Dammann, Carl: Antbropologisch-Etbnologisches Album in Photographien [...J. Berlin 1873/74. 


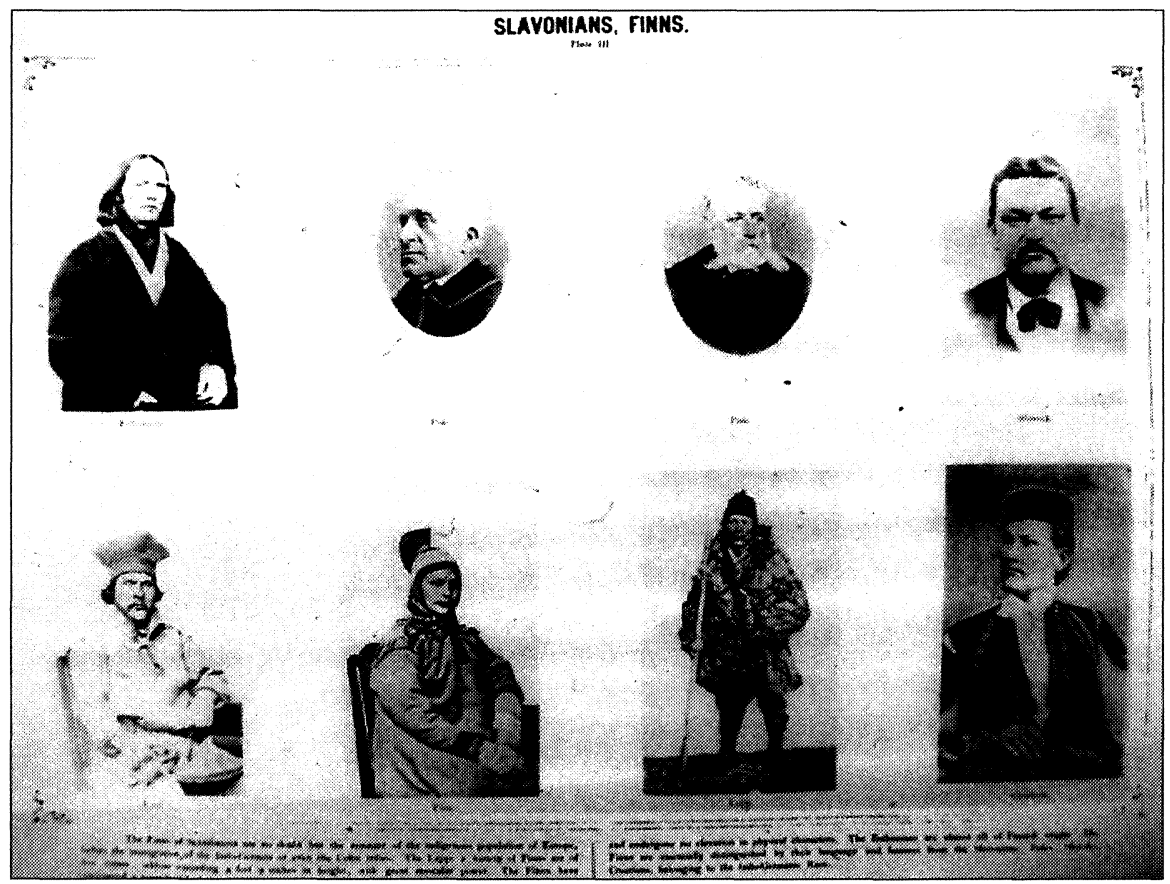

Wie auch schon die Types prinçipaux standen auch die zahllosen Visitkartenphotographien Dammanns räumlich, das heißt geographisch geordnet, nebeneinander auf den Tableaus. Damit verwies sein Anlageprinzip noch ganz auf die überkommene Systematik der Naturgeschichte als Verzeichnis alles Lebendigen zurück: "Das Klassifikationsraster der Naturgeschichte ist räumlich bestimmt, die Varietäten der Lebewesen werden in Form eines Tableaus dargestellt, das es [...] erlaubt, sowohl die Einheit der Menschengattung wie ihre Diversität zu belegen" ${ }^{102}$. Auf diese Vielfalt der menschlichen Erscheinungsformen, von Wolf Lepenies in seiner Studie Das Ende der Naturgeschichte, "Diversität" genannt, kam es seinerzeit Fritsch besonders an. Er lobte in einer Rezension Dammann gerade dafür, die "Seltensten Typen" ${ }^{103}$ zusammengebracht zu haben.

Allerdings geschah dies nicht in gedruckter Form, sondern mit einzeln aufgeklebten Photographien - mediengeschichtlich eine Zwischenerscheinung, die wegen des hohen Aufwandes und des exorbitanten Verkaufspreises keine Zukunft haben sollte. Das Album blieb ein Torso, denn das Erscheinen wurde nur kurze Zeit nach dem Tode des Herausgebers eingestellt. Spätere Publikationen wie etwa Ratzels von 1885 an erschienene

102 Lepenies (1976: 58).

103 Fritsch (1874: 68). 
dreibändige Völkerkunde sollten als Illustrationen dann eine Vielzahl photographischer Bildvorlagen in Gestalt von Xylographien wiedergegeben. Der gemeinsame Druck von Text und Bild erwies sich als der erfolgsträchtigere Weg zu einer illustrierten Völkerkunde.

Ein weiterer Grund für das Scheitern des Albums ist darin zu vermuten, daß es in seiner typologischen Konzeption allmählich unzeitgemäß wurde. Die physische Anthropologie wandte sich seit der Mitte des Jahrhunderts unter dem Eindruck der Evolutionstheorie immer stärker zeitlich geordneten Systematiken zu. Zahllose Funde in der Archäologie und Paläontologie hatten die Erkenntnis einer historischen Entwicklung der gesamten Natur und damit auch des Menschengeschlechtes bewirkt. An die Stelle einer Klassifizierung der Lebewesen als ein hierarchisch gegliedertes und durch die göttliche Schöpfung entstandenes zeitgleiches Nebeneinander sollte das Nacheinander einer allmählichen Anpassung und Differenzierung treten - von einfachen Formen hin zu immer komplexeren Gebilden. Statt eines Inventars der unhistorisch aufgefaßten Naturgeschichte mit starr abgegrenzten Arten ${ }^{104}$ sollten in phylogenetischen Systemen die Arten nicht länger mehr nach der Nähe oder Ferne zu einem ideellen Typus, sondern nach ihrer stammesgeschichtlichen Verwandtschaft und Abkunft von einem tatsächlichen Vorfabren angeordnet werden. Die Evolutionstheorie sollte hierin "Klassifikationen zu Genealogien werden lassen" ${ }^{105}$. Damit waren Herders "Naturvölker" für die Ethnologie, wie Lepenies dies zuspitzt, nicht länger mehr Brüder (Herder) oder Vettern (Lichtenberg), sondern fortan Großväter oder "zeitgenössische Vorfahren" ${ }^{106}$.

Für die sich seit den siebziger Jahren des 19. Jahrhunderts institutionalisierende Völkerkunde sollte Dammanns Album mit seiner bunten Vielfalt eher unspezifischer Atelieraufnahmen völlig unterschiedlicher Herkunft und Aufnahmeintentionen, nicht länger mehr eine ernstzunehmende wissenschaftliche Quelle sein. Bastian hatte dies 1872 in seiner Ankündigung des Werkes auch bereits selbst als möglichen Kritikpunkt erwähnt, nämlich die mangelnde Trennung in Aufnahmen zum Zwecke der physischen Anthropologie und in solche mit ethnographischen Intentionen: "Eine anfänglich beabsichtigte Scheidung zwischen anthropologischen und ethnologischen Darstellungen erwies sich nicht gut durchführbar, da bis jetzt bei den Aufnahmen diese Trennung nicht genugsam berücksichtigt wird, und deshalb zu viele der Bilder einen gemischten Charakter tragen ${ }^{107}$. Eine

\footnotetext{
104 Vgl. Lepenies (1976: 41-51).

105 Vgl. Lepenies (1980: 223).

106 Vgl. Lepenies (1976: 76f.).

107 Bastian (1872: 392).
} 
solche Unterscheidung gewann jedoch in den Aufnahmehinweisen Fritsch's von $1872{ }^{108}$ und 1875 an Konturen und wies den Weg zu einer systematischen visuellen Erfassung des Fremden.

\section{SCHLUß}

Mit dem Ende des 19. Jahrhunderts verschob sich das wissenschaftliche Interesse in der Ethnographie zusehends auf die sogenannten "Naturvölker, die häufig aus evolutionistischer Sicht als Überbleibsel früherer Entwicklungsstufen betrachtet wurden. Neben solche Kompilationen, wie etwa Ratzels Völkerkunde trat eine immer stärkere Konzentration der Forschung auf einzelne Ethnien: Monographisch berichtende, differenzierte Arbeiten zu Sprache und Sozialstruktur, zu Sitten und Gebräuchen, und dem materiellen Kulturinventar entstanden. Mit der Wende zum 20. Jahrhundert sollten eher kulturhistorische Fragestellungen die Forschung bestimmen, die Entstehung und Verbreitung von Kulturmerkmalen aber auch Abstammung, Wanderungsbewegungen und die Vermischung verschiedener Völker. Nicht mehr nur die große Gesamtschau der Schreibtischgelehrten, sondern der detaillierte Bericht einzelner Forschungsreisender sowohl über das körperliche Erscheinungsbild als auch über Gebräuche, das Vokabular oder die Dokumentation von Ethnographika sollten in den Mittelpunkt des Interesses rücken. Zugleich verlagerte sich die visuelle ethnographische Dokumentation hin zu den Reisenden selbst. Mit dem Aufkommen der teilnehmenden Beobachtung bei stationären Feldforschungsaufenthalten, medientechnischen Innovationen und der Entwicklung des modernen Bildjournalismus sind weitere Schlagworte genannt, welche die Medienpraxis der Forschungsreisenden im 20. Jahrhundert prägen sollten.

\section{LITERATURVERZEICHNIS}

Allgemeine deutsche Real-Encyklopädie für die gebildeten Stände. Conversations-Lexikon, 1836: Band 11, Leipzig, zitiert als Brockhaus.

ANONYMus, 1845: "Anwendung der Photographie zum Studium der Menschenraçen", Polytechnisches Journal, 26. H. 17: 400.

108 So hieß es in den Rathschlägen (1872:28): "Man hat zu unterscheiden zwischen physiognomischen Aufnahmen und ethnographischen. Die Grundprincipien, nach welchen beide Arten von Aufnahmen anzufertigen sind, müssen getrennt werden." Hervorhebungen ebd. 
BaER, Karl Ernst von: Types Prinçipaux Des Différentes Raçes Humaines dans les cinq parties Du Monde, modélés sous la direction du Pr. Baer de St. Petersbourg. Ohne Ort und Jahr.

- 1861: Typen von National-Physiognomien aus Steinpappe, Besonderer Abdruck aus No. 132 der deutschen St. Petersburger Zeitung.

BAER, Karl Ernst von und Rudolph WAGNER, 1861: Bericht über die Zusammenkunft einiger Anthropologen im September 1861 in Göttingen zum Zwecke gemeinsamer Besprechungen, Leipzig.

[BASTIAN, Adolf], 1869: Miszelle o. T., Zeitschrift für Ethnologie, 1: 399f..

B.[ASTIAN, Adolf], 1872: Miszelle o. T., Zeitschrift für Ethnologie, 4 :392.

Dr. B.[ASTIAN, Adolf], 1873: “Die Photographie im Dienste der Geographie und Anthropologie", Globus, 24: 10-12.

BASTIAN, Adolf, 1869: "Das natürliche System in der Ethnologie", Zeitschrift für Ethnologie, 1: 1-23.

- 1875: Schöpfung oder Entstehung. Aphorismen zur Entwicklung des organischen Lebens, Jena.

BITTERLI, Urs, 1982: Die "Wilden" und die "Zivilisierten": Die europäisch-überseeische Begegnung, München [1976].

BLUMENBACH, Johann Friedrich, 1796-1810: Abbildungen naturbistorischer Gegenstände. 10 Hefte mit 100 Tafeln, Göttingen.

Dammann, Carl, 1873-74: Anthropologisch-Etbnologisches Album in Photographien. Herausgegeben mit Unterstützung aus den Sammlungen der Berliner Gesellschaft für Anthropologie, Ethnologie und Urgeschichte, Berlin.

DougherTY, Frank W. P., 1990: "Christoph Meiners und Johann Friedrich Blumenbach im Streit um den Begriff der Menschenrassen, Soemmering-Forschungen. Band 6. "Die Natur des Menschen: Probleme der Physischen Anthropologie und Rassenkunde (1750-1850)", Mainz-Stuttgart-New York: 89-111.

EDWARDS, Elizabeth, 1990: "Photographic "Types": The Pursuit of Method", Visual Anthropology, 3: 235-258.

FALCONER, John, 1984-86: “Ethnographical Photography in India 1850-1900", Photographic Collector, 5: 16-46.

FIEDERMUTZ-LAUN, Annemarie, 1970: Der kulturhistorische Gedanke bei Adolf Bastian, Wiesbaden.

FISCHER, Hans, 1983: "Anfänge, Abgrenzungen, Anwendungen", in Ders (hg.), Etbnologie. Eine Einfübrung, Berlin: 11-46.

FriTsCH, Gustav, 1875: "Praktische Gesichtspunkte für die Verwendung zweier dem Reisenden wichtigen technischen Hülfsmittel: das Mikroskop und der photographische Apparat,, in Neumayer (hg.), 1875: 591-625.

- 1881: "Sonst und Jetzt der menschlichen Rassenkunde vom morphologischen Standpunkt", in Verhandlungen der Berliner Gesellschaft für Anthropologie, Ethnologie und Urgeschichte, Zeitschrift für Ethnologie, 13: 210-217.

- 1911: "Anthropologie", in K. W. Wolf-Czapek, Angewandte Photographie in Wissenschaft und Technik. Band 4, Berlin: 17-34.

GalTON, Francis, 1878: "Composite Portraits made by combining those of many different persons into a single resultant figure", Nature, 18: 97-100.

GOETHE, Johann Wolfgang von, 1969: Schriften zur Morphologie II. Hg. v. Wilfried Malsch, in Gesamtausgabe der Werke und Schriften in zweiundzwanzig Bänden, Stuttgart. 
GreBe, Paul (bearb.), 1963: Duden. Band 7. Etymologie, Darmstadt.

Grimm, Jacob und Wilhelm, 1952: Deutsches Wörterbuch. Band 11. I, 2, Leipzig.

H.[ARTMANN, Robert], 1869: O. T. [Mitteilung über John Lampreys "Plan zur Veranstaltung von Messungen photographisch aufgenommener Individuen"], Zeitschrift für Ethnologie, 1: 184.

HERDER, Johann Gottfried, 1887: Ideen zur Philosophie der Geschichte der Menschbeit. Erster und zweiter Teil. [1784, 1785], in J. G. Herder, Sämtliche Werke. Hg. von Bernhard Suphan. Band XIII, Berlin. Reprint: Hildesheim 1967.

HildEBRANDT, Hans-Jürgen, 1994: "Thesen zur Geschichte der Ethnologie", in Matthias S. Laubscher \& Bertram Turner (hg.), Völkerkunde-Tagung 1991. Band 1. Systematische Völkerkunde, München: 347-359.

HOCHSTETTER, Ferdinand von, 1863: Neu-Seeland, Stuttgart.

KICK, Wilhelm, 1982: "Alexander von Humboldt und die Brüder Schlagintweit", in Claudius C. Müller \& Walter Raunig (hg.), Der Weg zum Dach der Welt, Innsbruck: 75-77.

KöpPING, Klaus-Peter, 1983: Adolf Bastian and the Psychic Unity of Mankind: The Foundation of Anthropology in Nineteenth Century Germany, St. Lucia-London-New York.

KÖRNER, Hans, 1989: "Photographieren auf Forschungsreisen. Robert Schlagintweit und seine Brüder erforschen die Alpen, Indien und Hochasien", in Bodo von Dewitz \& Reinhard Matz (hg.), Silber und Salz: Zur Frühzeit der Photographie im deutschen Sprachraum 1839-1860, Köln-Heidelberg: 310-320.

KoHL, Karl-Heinz, 1993: Etbnologie - die Wissenschaft vom kulturell Fremden. Eine Einfübrung, München.

KrauSE, Rudolf, 1881: "II. Anthropologische Abtheilung", in Johannes Dietrich Eduard Schmeltz \& Rudolf Krause, Die ethnographisch-antbropologische Abtheilung des Museum Godeffroy in Hamburg. Ein Beitrag zur Kunde der Südsee-Völker, Hamburg: 503-670.

KRECH, Hartmut, 1984: "Lichtbilder vom Menschen. Vom Typenbild zur anthropologischen Fotografien, Fotogeschichte, 4. H. 14: 3-15.

LAMPREY, John, 1869: "On a Method of Measuring the Human Form for Students of Ethnology", Journal of the Ethnological Society of London, 1: $84 \mathrm{f}$.

LEPENIES, Wolf, 1976: Das Ende der Naturgeschichte: Wandel kultureller Selbstverständlichkeiten in den Wissenschaften des 18. und 19. Jabrbunderts, München.

- 1980: "Naturgeschichte und Anthropologie im 18. Jahrhundert", in Bernhard Fabian, Wilhelm Schmidt-Biggemann \& Rudolf Vierhaus, Deutschlands kulturelle Entfaltung - Die Neubestimmung des Menschen. Studien zum 18. Jabrbundert. Band 2/3, München: 211-226.

LISSAUER, Abraham, 1905: "Ansprache des Vorsitzenden der Berliner Anthropologischen Gesellschaft [zum Tode Adolf Bastians]", Zeitschrift für Ethnologie, 37: 234-236.

MAYR, Ernst, 1990: "Die Darwinsche Evolution und die Widerstände gegen die Selektionstheorie", in Jost Herbig \& Rainer Hohlfeld (hg.), Die Zweite Schöpfung: Geist und Ungeist in der Biologie des 20. Jabrbunderts, München: 44-70.

MeITZEN, August, 1875: "Politische Geographie und Statistik", in Neumayer (hg.), op. cit.: $151-172$

MEYER-ABICH, Adolf, 1949: Biologie der Goethezeit, Stuttgart.

Meyers Konversations-Lexikon. Eine Encyklopädie des allgemeinen Wissens, Band 15, Leipzig, $1878^{3}$.

MÜHLMANN, Wilhelm E., 1984: Geschichte der Anthropologie, Wiesbaden, 3." ed. 
MÜllER, Friedrich, 1873: Allgemeine Etbnographie, Wien.

NEUMAYER, Georg von (hg.), 1875: Anleitung zu wissenschaftlichen Beobachtungen auf Reisen. Mit besonderer Rücksicht auf die Bedürfnisse der kaiserlichen Marine, Berlin.

PESCHEL, Oscar, 1865: Geschichte der Erdkunde bis auf A. v. Humboldt und Carl Ritter, München.

- 1875: Völkerkunde, Leipzig, 2:" ed.

Ratbschläge für antbropologische Untersuchungen auf Expeditionen der Marine. Auf Veranlassung des Chefs der Kaiserlich Deutschen Admiralität ausgearbeitet von der Berliner Gesellschaft für Anthropologie, Ethnologie und Urgeschichte, Berlin, 1872.

RATZEL, Friedrich, 1885-86-88: Völkerkunde. 3 Bände, Leipzig.

ROSENBERG, Gerd, 1984: Wilbelm Burger: Ein Welt- und Forschungsreisender mit der Kamera 1844-1920, Wien-München.

SCHERZER, Karl von und Eduard SCHWARZ, 1859: “Ueber Körpermessungen, als Behelf zur Diagnostik der Menschenracen. Entwurf eines Systems, welches die Verfasser, den von ihnen, während der Reise der k. k. Österreichischen Fregatte Novara um die Erde, an Individuen verschiedener Racen angestellten Messungen zu Grunde gelegt haben. [...] am Bord Sr. Majestät Fregatte Novara, 7. October 1858", Mittbeilungen der Kaiserlich-Königlichen Geograpbischen Gesellschaft, 3: 11-31.

Schulz, Hans (Begr.), 1981: Deutsches Fremdwörterbuch. Band 5, Berlin-New York.

SERRES, Etienne-Renaud-Augustin, 1843: O. T. Présentation de "cinq portraits représentant deux naturels de l'Amérique du Sud (Botocudes)", Comptes Rendus de l'Académie des Sciences, 19: 490.

- 1845: "Observations sur l'application de la photographie à l'étude des races humaines". In: Comptes Rendus de l'Académie des Sciences, 21: 242-246.

STEINTHAL, Heymann (Heinrich), 1875: "Linguistik", in Neumayer (hg.), op. cit.: 551-570.

STENGER, Erich, 1938: Die Photographie in Kultur und Technik: Ibre Geschichte während bundert Jabren, Leipzig.

STRECK, Bernhard, 1987: Wörterbuch der Etbnologie, Köln.

THEYE, Thomas (hg.), 1989: Der geraubte Schatten. Photograpbie als ethnographisches Dokument, München-Luzern. Darin: "Wir wollen nicht glauben, sondern schauen”. Zur Geschichte der ethnographischen Fotografie im deutschsprachigen Raum im 19. Jahrhundert": 60-72, 89-119.

- 1995: "Licht- und Schattenbilder aus den Binnenlanden von Java": Franz Wilhelm Junghuhn, Karl Ritter von Scherzer und die Erdumsegelung der K. K. Fregatte "Novara" in den Jahren 1857 bis 1859", Fotogeschichte, 15, H. 56: 3-22.

VERMEUlen, Han F., 1995: "Origins and Institutionalization of Ethnography and Ethnology in Europe and the USA, 1771-1845", in Han F. Vermeulen \& Arturo Álvarez Roldán (hg.), Fieldwork and Footnotes: Studies in the History of European Anthropology, London-New York: 39-59.

VIRCHOw, Rudolf, 1875: "Anthropologie und prähistorische Forschungen", in Neumayer (hg.), op. cit.: 571-590.

VOGT, Carl, 1863: Vorlesungen ïber den Menschen, seine Stellung in der Schöpfung und in der Geschichte der Erde. 2 Bände, Giessen.

WEIGAND, Friedrich L. K., 1910: Deutsches Wörterbuch. Band 2, Gießen, 5." ed. Reprint: Berlin 1968.

ZEDLER, Johann Heinrich, 1982: Grosses Vollständiges Universal-Lexikon. Band 50, Leipzig/Halle 1746. Reprint: Graz 1982. 
En el ámbito germano-parlante se intentó documentar las manifestaciones corporales y culturales del hombre a través de fotografías, como medio de inventario visual, incidiendo más en la antropología física que en la etnografía. Entre los diferentes intentos para la creación de una tipología morfológica, la cuestionable visión de Johann Goethe pasó a ser la sistematización conceptual dominante frente a otros modelos empíricamente fundados. El concepto de un museo fotográfico de las razas humanas constituyó la idea central para una aplicación científica de la fotografía. Su utilización se acrecentó a fines del siglo XIX, tanto en la ilustración de compilaciones de carácter global, como en representaciones de grupos étnicos completos. Las innovaciones técnicas fomentaron las iniciativas tendentes a superar la estática fotografía habitual.

In the German-speaking world, attempts were made to document Man's bodily and cultural manifestations by means of photographs. Photography thus became a medium for making visual inventories, its impact being larger in physical anthropology than in ethnography. Of the steps taken to establish a morphological typology, that of Johann Goethe, a questionable conceptual systematization, prevailed over other empirically-based models. Late in the 19th century, the idea of a Photographic Museum of the Human Races lay behind the ever more frequent scientific application of photography to illustrations in compilations of a general character and to representations of whole ethnic groups. Technical innovations promoted initiatives aimed to overcome the static photography of the day. 